\title{
Radio Sources in the 2dF Galaxy Redshift Survey I. Radio Source Populations*
}

\author{
Elaine M. Sadler ${ }^{1}$, V. J. McIntyre ${ }^{1}$, \\ C. A. Jackson ${ }^{1}$ and R. D. Cannon ${ }^{2}$ \\ ${ }^{1}$ School of Physics, University of Sydney, NSW 2006, Australia \\ ems@physics.usyd.edu.au,vjm@physics.usyd.edu.au, cjackson@physics.usyd.edu.au \\ ${ }^{2}$ Anglo-Australian Observatory, PO Box 296, Epping, NSW 2121, Australia \\ rdc@aaoepp.aao.gov.au \\ Received 1999 May 27, accepted 1999 August 31
}

\begin{abstract}
We present the first results from a study of the radio continuum properties of galaxies in the 2dF Galaxy Redshift Survey, (2dFGRS) based on thirty 2dF fields covering a total area of about $100 \mathrm{deg}^{2}$. About $1.5 \%$ of galaxies with $b_{\mathrm{J}}<19 \cdot 4$ mag. are detected as radio continuum sources in the NRAO VLA Sky Survey (NVSS). Of these, roughly $40 \%$ are star-forming galaxies and $60 \%$ are active galaxies (mostly low-power radio galaxies and a few Seyferts). The combination of 2dFGRS and NVSS will eventually yield a homogeneous set of around 4000 radio-galaxy spectra, which will be a powerful tool for studying the distribution and evolution of both AGN and starburst galaxies out to $z \sim 0 \cdot 3$.
\end{abstract}

Keywords: radio continuum: galaxies — galaxies: distances and redshifts — galaxies: active - galaxies: starburst

\section{Introduction}

A new generation of sensitive large-area radiosource surveys at milliJanksy levels $\left(1 \mathrm{Jy}=10^{-26}\right.$ $\mathrm{W} \mathrm{m} \mathrm{m}^{-2} \mathrm{~Hz}^{-1}$ ) is now becoming available. They include the FIRST survey (Becker, White \& Helfand 1995), WENSS (Rengelink et al 1997), NVSS (Condon et al. 1998) and SUMSS (Bock, Large \& Sadler 1999). These surveys offer some important advantages for cosmological studies - they reach sufficiently high source densities that detection of large-scale structure is possible (Cress et al. 1996; Magliocchetti et al. 1998), and also probe a second cosmologically-significant radio source population, that of star-forming galaxies, which are rarely seen in strong-source surveys.

Deep radio surveys of a few small areas of sky at 1. $4 \mathrm{GHz}$ (Condon 1984; Windhorst et al. 1985; see also Condon 1992) have shown that there are two distinct populations of extragalactic radio sources. Over $95 \%$ of radio sources above about $50 \mathrm{mJy}$ are classical radio galaxies and quasars (median redshift $z \sim 1$ ) powered by active galactic nuclei (AGN), while the remainder are identified with star-forming galaxies (median $z \sim 0 \cdot 1$ ). The fraction of starforming galaxies increases rapidly below $10 \mathrm{mJy}$, and below $1 \mathrm{mJy}$ they begin to be the dominant population.

The scientific return from radio continuum surveys is enormously increased if the optical counterparts of the radio sources can be identified and their redshift distribution measured. In the past, this has been a slow and tedious process which could only be carried out for relatively small samples. However, the Anglo-Australian Observatory's Twodegree Field ( $2 \mathrm{dF}$ ) spectrograph now makes it possible to carry out spectroscopy of several hundred galaxies simultaneously. Here, we describe the first step in this process - the identification of faint radio-source counterparts among galaxies whose spectra have been obtained in the 2dF Galaxy Redshift Survey.

The 2dFGRS (Colless 1999; Maddox 1998) is a large-scale survey of 250,000 galaxies covering 2000 square degrees in the southern hemisphere. The survey is designed to be almost complete down to a limiting apparent magnitude of $b_{\mathrm{J}}=19 \cdot 4$. The median redshift of the galaxies is about $0 \cdot 1$

\footnotetext{
* Based on data obtained by the 2dFGRS Team: J Bland-Hawthorn (AAO), R D Cannon (AAO), S Cole (Durham), M Colless (ANU, Australian convenor), C A Collins (Liverpool J Moores), W J Couch (UNSW), N Cross (St Andrews), G B Dalton (Oxford), K E Deeley (UNSW/AAO), R De Propris (UNSW), S P Driver (St Andrews), G Efstathiou (Cambridge), R S Ellis (Cambridge, UK convenor), S Folkes (Cambridge), C S Frenk (Durham), K Glazebrook (AAO), N J Kaiser (Hawaii), O Lahav (Cambridge), I J Lewis (AAO), S L Lumsden (Leeds), S J Maddox (Cambridge), S Moody (Cambridge), P Norberg (Durham), J A Peacock (Edinburgh), B A Peterson (ANU), I A Price (ANU), S Ronen (Cambridge), M Seabourne (Oxford), R Smith (Edinburgh), W J Sutherland (Oxford), H Tadros (Oxford) and K Taylor (AAO).
} 


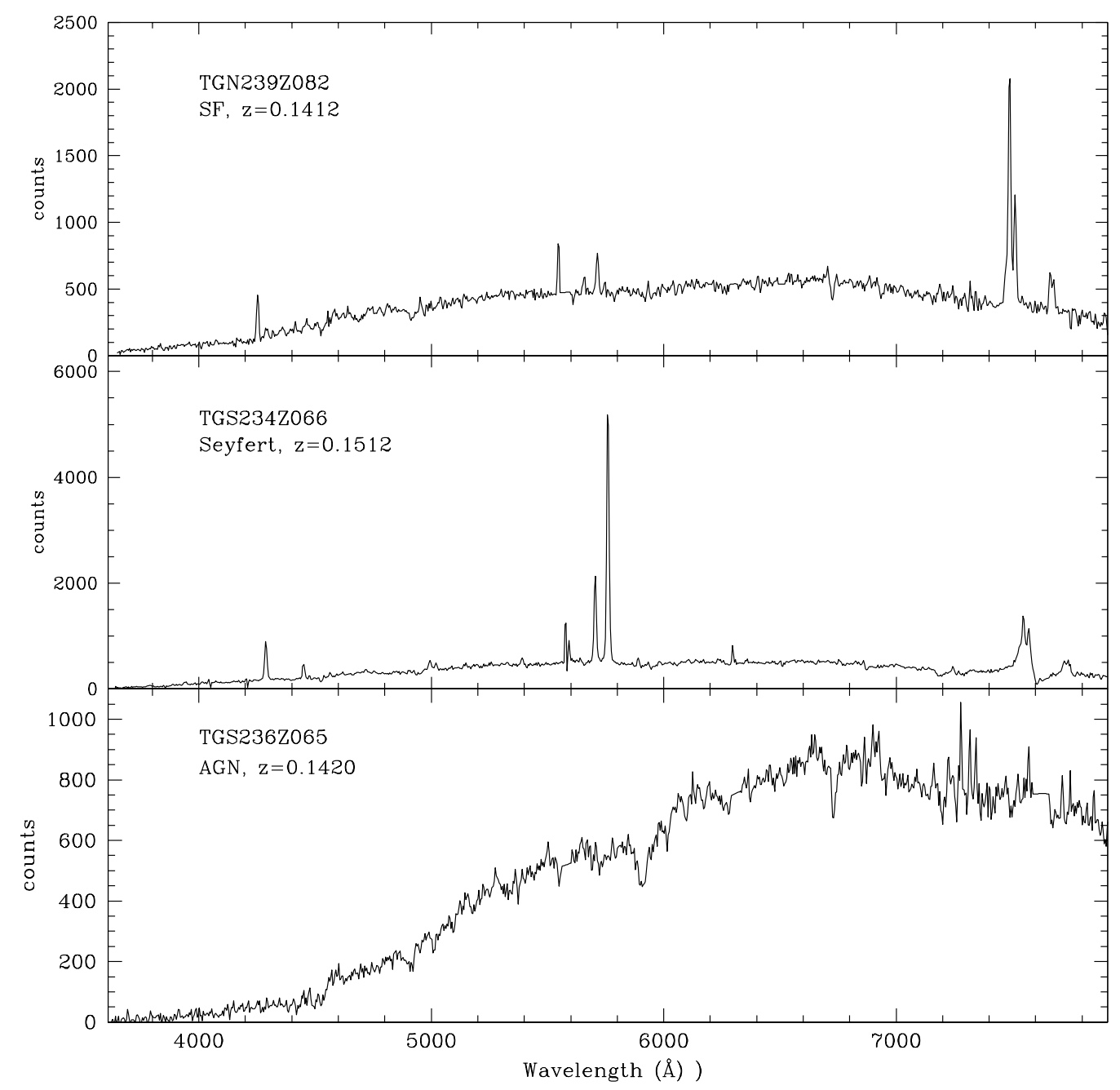

Figure 1-Spectra of three 2dFGRS radio sources, showing the three main spectral classes: (top) A star-forming galaxy, TGN239Z082, with strong Balmer emission lines of $\mathrm{H} \alpha \lambda 6563$ and $\mathrm{H} \beta \lambda 4861$, as well as weaker emission lines of [SII] $\lambda 6716,6731,[\mathrm{NII}] \lambda 6583$, [OIII] $\lambda 4959,5007$ and [OII] $\lambda 3727 ;$ (middle) an emission-line AGN, TGS234Z066, with a Seyfert-like spectrum which has broad $\mathrm{H} \alpha$ emission and [OIII] much stronger than $\mathrm{H} \beta$; and (bottom) A radio galaxy, TGS236Z065, with an absorption-line spectrum typical of giant elliptical galaxies.

and the great majority have $z<0 \cdot 3$. Spectra are being obtained using the $2 \mathrm{dF}$ multi-object fibre optic spectroscopic system on the $3.9 \mathrm{~m}$ AngloAustralian Telescope (AAT) (Lewis, Glazebrook \& Taylor 1998; Smith \& Lankshear 1998). The 2dF enables the AAT to obtain spectra for 400 objects simultaneously, spread over a field which is two degrees in diameter. The survey will cover two large strips of sky at high Galactic latitude, one each in the southern and northern Galactic hemispheres, plus outlying random fields in the south. The first test data were obtained in 1997 and the survey is expected to be substantially complete by the end of 2000 .

This paper presents the first results from what will eventually be a much larger study. When the 2dFGRS is complete, it will yield around 4000 good-quality spectra of galaxies associated with faint radio sources - by far the largest sample of radio-galaxy spectra ever compiled. Our aim in this paper is to present a first, qualitative exploration of the faint radio source population as observed by $2 \mathrm{dF}$ and the NVSS.

Throughout this paper, we use $H_{0}=75 \mathrm{~km} \mathrm{~s}^{-1}$ $\mathrm{Mpc}^{-1}$ and $q_{0}=0 \cdot 5$.

\section{The Optical Data}

Our data set comprises the thirty fields observed by the 2dFGRS team in November 1997 and January 1998. Twenty-three of these are in the southern Galactic hemisphere and the other seven in the north. They include a total of 8362 target galaxies brighter than $b_{\mathrm{J}}$ magnitude $19 \cdot 4$.

Although each $2 \mathrm{dF}$ field covers about $3 \cdot 14$ square degrees, the total effective area covered by the 30 fields is somewhat less than 90 square degrees because there is some overlap between adjacent fields. Also, the fractional completeness of this early sample varies substantially from field to field. In many fields about $30 \%$ of the fibres were allocated to targets in a parallel QSO survey; the 2dFGRS uses a flexible tiling algorithm to deal with this 
and with the intrinsic variations in target density across the sky. When complete, the GRS will yield spectra for around $95 \%$ of all galaxies in the input catalogue. The current sample has variable levels of completeness (in terms of the surface distribution of all galaxies brighter than $b_{\mathrm{J}}=19 \cdot 4 \mathrm{mag}$.), though there should be no systematic effects depending on the magnitude, redshift or other properties of the galaxies.

The standard observing pattern for the 2dFGRS is a set of three consecutive 20-minute exposures per field, together with calibration arcs and flat fields. The total exposure time is well-matched to the time required to reconfigure a second set of fibres for the next observation. Up to 380 galaxies can be observed simultaneously, with some 20 fibres allocated to sky. In many fields, however, the total number of galaxies is closer to 250 since a deep QSO survey is being carried out in parallel with the galaxy redshift survey.

Using gratings of 300 lines per mm, the 2dFGRS spectra cover the wavelength range $3800 \AA$ to 8000 $\AA$ at a resolution of about $10 \AA$. Most spectra have a signal-to-noise ratio $(S / N)$ of 10 (per $4 \AA$ pixel) or better. Reliable redshifts are obtained for up to $95 \%$ of the targets in good observing conditions; the survey average is currently about 90\% (Folkes et al. 1999). About 5\% of the targets are found to be foreground stars; the original selection was for objects which appeared non-stellar in digitised data from UK Schmidt Telescope sky survey photographs, using a conservative criterion to minimise the number of galaxies missed.

Figure 1 shows three spectra from the survey, and gives an idea of the typical quality of 2dFGRS spectra for galaxies with redshifts around $z=0 \cdot 15$ and $b_{\mathrm{J}}$ magnitudes in the range $17 \cdot 6-18 \cdot 9$.

\section{The Radio Data}

The NRAO VLA Sky Survey (NVSS; Condon et al. $1998)$ is a $1.4 \mathrm{GHz}(20 \mathrm{~cm})$ radio imaging survey of the entire sky north of declination $-40^{\circ}$. The survey catalogue contains sources as weak as 2.5 $\mathrm{mJy}$, and is essentially complete above $3.5 \mathrm{mJy}$.

We used the NVSS source catalogue to identify candidate radio-emitting galaxies in the 2dFGRS. Subsets of the NVSS catalogue were extracted to match the RA and Dec range covered by each of the $2 \mathrm{dF}$ fields. The NVSS source density is roughly 60 per square degree, so each of these sub-catalogues contained about 200 radio sources. At this stage, we did not attempt to identify 'double' NVSS sources. However, we estimate [using an algorithm similar to that adopted by Magliocchetti et al. (1998) for identifying double radio sources in the FIRST survey] that these represent only a small fraction of NVSS sources (of order 1\%), so the presence of double sources does not significantly affect our results in this small sample.

We compared the NVSS and 2dFGRS catalogues for each field and identified the galaxies for which there is a candidate radio 'match', i.e. an NVSS radio source lying within 15 arcsec of the position of a $2 \mathrm{dF}$ galaxy. The 15 arcsec limit was chosen because our earlier Monte Carlo tests using the COSMOS database suggested that most candidate matches of bright galaxies (i.e. $b_{\mathrm{J}}<19 \cdot 4 \mathrm{mag}$.) with radio-optical separation up to 10 arcsec are real associations, together with a substantial fraction of those with offsets of $10-15$ arcsec.

The uncertainty in the NVSS radio source positions increases from a $1 \sigma$ error of $1-2$ arcsec at 10 mJy to 4-5 arcsec (and occasionally up to 10 arcsec) for the faintest (2-3 mJy) sources (Condon et al. 1998). In addition, centroiding of bright radio sources with extended radio structure can be somewhat uncertain and may make optical identification difficult. Determining the optical centroid also becomes imprecise for large nearby galaxies. In these cases, overlaying the radio contours on an optical image usually makes it clear whether or not the candidate ID is correct.

We found a total of 127 candidate matches in the 30 fields studied, i.e. $1.5 \%$ of the $83622 \mathrm{dF}$ galaxies in the survey area. Of these, 99 had radio-optical offsets of less than 10 arcsec.

We also ran the matching program twice more, offsetting all the radio positions by 3 and 5 arcmin. Any matches produced from this 'off-source' catalogue should be chance coincidences, allowing us to estimate the number of matches expected purely by chance. Table 1 lists the results of this test, and shows the distribution of the offset $D$ (the difference between radio and optical positions) for matches found in the 'on-source' and 'off-source' tests.

Table 1. Comparison of on-source and off-source matches as a function of radio-optical offset $D$

'Off-source' data are for matches where the radio-source positions were offset in declination by 3 arcmin (Off 3 ) and 5 arcmin (Off 5)

\begin{tabular}{cccccc}
\hline$D$ & \multicolumn{2}{c}{ No. of matches } & $\begin{array}{c}\text { Predicted } \\
\text { counts }\end{array}$ & $\begin{array}{c}\text { Probability that } \\
\text { match is real }\end{array}$ \\
\hline $0-2 \cdot 5$ & 34 & 3 & 0 & 1 & $96 \%$ \\
$2 \cdot 5-5 \cdot 0$ & 29 & 2 & 1 & 2 & $95 \%$ \\
$5 \cdot 0-10 \cdot 0$ & 36 & 6 & 8 & 9 & $81 \%$ \\
$10 \cdot 0-15 \cdot 0$ & 28 & 12 & 13 & 15 & $55 \%$ \\
\hline
\end{tabular}

A further check comes from predicting the expected number of chance coincidences, based on the average surface densities of objects. Since 15 arcsec is $1 / 240$ of the 1 degree radius of each $2 \mathrm{dF}$ field, and there are about 60 NVSS sources per square degree, the chance that a given GRS target will fall within 15 arcsec of an unrelated radio source is about $60 \times \pi / 240^{2}$ (the resolution of the NVSS is such that at most one 
Table 2. Galaxies with $\Delta<10^{\prime \prime}$ and good-quality spectra

\begin{tabular}{|c|c|c|c|c|c|c|c|}
\hline Field & ID & $\begin{array}{c}\text { Offset } \\
\text { (arcsec) }\end{array}$ & $\begin{array}{c}B(J) \\
\text { (mag) }\end{array}$ & $z$ & $\begin{array}{c}S_{1 \cdot 4} \\
(\mathrm{mJy})\end{array}$ & $\begin{array}{c}\text { IRAS } \\
S_{60}(\mathrm{Jy})\end{array}$ & $\begin{array}{l}\text { Spectral } \\
\text { class }\end{array}$ \\
\hline $166 \mathrm{~s}$ & TGS166Z108 & $6 \cdot 7$ & $17 \cdot 2$ & $0 \cdot 0801$ & $3 \cdot 0$ & $0 \cdot 297$ & $\mathrm{SF}$ \\
\hline $203 \mathrm{~s}$ & TGS203Z019 & $2 \cdot 4$ & $18 \cdot 9$ & $0 \cdot 2358$ & $85 \cdot 1$ & & AGN(abs) \\
\hline $203 \mathrm{~s}$ & XGS202Z238 & $0 \cdot 9$ & $18 \cdot 1$ & $0 \cdot 1389$ & $90 \cdot 5$ & & AGN(abs) \\
\hline $204 \mathrm{~s}$ & TGS204Z102 & $1 \cdot 8$ & $16 \cdot 8$ & $0 \cdot 0759$ & $3 \cdot 2$ & & AGN(abs) \\
\hline $204 s$ & TGS204Z037 & $1 \cdot 8$ & $19 \cdot 4$ & $0 \cdot 2643$ & $54 \cdot 0$ & & $\mathrm{AGN}(\mathrm{E}+[\mathrm{OIII}] \mathrm{em})$ \\
\hline $206 \mathrm{~s}$ & TGS206Z164 & $8 \cdot 8$ & $14 \cdot 6$ & $0 \cdot 0255$ & $2 \cdot 9$ & $0 \cdot 293$ & $\mathrm{SF}$ \\
\hline $206 \mathrm{~s}$ & TMS206Z015 & $1 \cdot 2$ & $17 \cdot 4$ & $0 \cdot 0700$ & $11 \cdot 3$ & $4 \cdot 294$ & $\mathrm{SF}$ \\
\hline $206 s$ & XGS284Z002 & $3 \cdot 7$ & $17 \cdot 7$ & $0 \cdot 0988$ & $7 \cdot 6$ & & AGN(abs) \\
\hline $207 \mathrm{~s}$ & XGS206Z038 & $0 \cdot 9$ & $19 \cdot 2$ & $0 \cdot 2424$ & $203 \cdot 4$ & & AGN(abs) \\
\hline $207 \mathrm{~s}$ & XGS208Z145 & $5 \cdot 2$ & $14 \cdot 1$ & $0 \cdot 0229$ & $4 \cdot 7$ & 0.597 & $\mathrm{SF}$ \\
\hline $207 \mathrm{~s}$ & TGS207Z011 & $0 \cdot 2$ & $14 \cdot 2$ & $0 \cdot 0355$ & $55 \cdot 0$ & & $\mathrm{AGN}(\mathrm{em})$ \\
\hline $218 \mathrm{~s}$ & XGS217Z158 & $3 \cdot 4$ & $14 \cdot 4$ & $0 \cdot 0207$ & $2 \cdot 5$ & $0 \cdot 416$ & $\mathrm{SF}$ \\
\hline $218 \mathrm{~s}$ & TGS218Z173 & $3 \cdot 4$ & $18 \cdot 6$ & $0 \cdot 0914$ & $8 \cdot 9$ & & $\mathrm{AGN}(\mathrm{em})$ \\
\hline $218 s$ & XGS219Z089 & $4 \cdot 5$ & $18 \cdot 7$ & $0 \cdot 2497$ & $59 \cdot 7$ & & AGN(abs) \\
\hline $219 \mathrm{~s}$ & TGS219Z142 & $7 \cdot 3$ & $17 \cdot 1$ & $0 \cdot 1245$ & $8 \cdot 5$ & & AGN(abs) \\
\hline 220 s & XGS221Z290 & $5 \cdot 1$ & $19 \cdot 1$ & $0 \cdot 2480$ & $7 \cdot 7$ & & $\mathrm{AGN}(\mathrm{E}+[\mathrm{OIII}] \mathrm{em})$ \\
\hline 220 s & TGS220Z015 & $0 \cdot 8$ & $19 \cdot 3$ & $0 \cdot 2630$ & $12 \cdot 5$ & & $\mathrm{AGN}(\mathrm{E}+[\mathrm{OIII}] \mathrm{em})$ \\
\hline $220 \mathrm{~s}$ & TGS220Z009 & $7 \cdot 9$ & $18 \cdot 7$ & $0 \cdot 2306$ & $3 \cdot 1$ & & $\mathrm{AGN}(\mathrm{E}+[\mathrm{OII}] \mathrm{em})$ \\
\hline $220 s$ & TGS220Z022 & $2 \cdot 8$ & $15 \cdot 6$ & $0 \cdot 0575$ & $6 \cdot 4$ & 0.574 & $\mathrm{SF}$ \\
\hline $220 s$ & TGS220Z097 & $3 \cdot 0$ & $14 \cdot 3$ & $0 \cdot 0204$ & $5 \cdot 4$ & $0 \cdot 953$ & $\mathrm{SF}$ \\
\hline 220 s & TGS220Z128 & $6 \cdot 7$ & $18 \cdot 9$ & $0 \cdot 1306$ & $3 \cdot 2$ & & AGN(em) \\
\hline $232 \mathrm{~s}$ & TGS232Z060 & $7 \cdot 6$ & $16 \cdot 8$ & $0 \cdot 0590$ & $3 \cdot 2$ & $0 \cdot 324$ & $\mathrm{SF}$ \\
\hline $232 \mathrm{~s}$ & TGS232Z156 & $5 \cdot 1$ & $19 \cdot 3$ & $0 \cdot 2446$ & $6 \cdot 1$ & & AGN(abs) \\
\hline $233 \mathrm{~s}$ & XMS232Z027 & $6 \cdot 3$ & $13 \cdot 2$ & $0 \cdot 0066$ & $9 \cdot 8$ & $2 \cdot 229$ & $\mathrm{SF}$ \\
\hline $233 \mathrm{~s}$ & TGS233Z084 & $7 \cdot 6$ & $18 \cdot 4$ & $0 \cdot 0648$ & $3 \cdot 8$ & & AGN?([OIII]em) \\
\hline $233 \mathrm{~s}$ & TGS233Z196 & $7 \cdot 2$ & $18 \cdot 3$ & $0 \cdot 1030$ & $2 \cdot 2$ & & $\mathrm{AGN}(\mathrm{abs})$ \\
\hline $234 \mathrm{~s}$ & TGS234Z066 & $1 \cdot 8$ & $18 \cdot 9$ & $0 \cdot 1512$ & $25 \cdot 2$ & & $\operatorname{AGN}(\mathrm{em})$ \\
\hline $234 \mathrm{~s}$ & TGS234Z197 & $6 \cdot 1$ & $17 \cdot 9$ & $0 \cdot 1092$ & $21 \cdot 1$ & & $\mathrm{AGN}(\mathrm{abs})$ \\
\hline $234 \mathrm{~s}$ & TGS234Z186 & $7 \cdot 4$ & $18 \cdot 0$ & $0 \cdot 1393$ & $4 \cdot 6$ & & $\mathrm{AGN}(\mathrm{abs})$ \\
\hline $234 \mathrm{~s}$ & TGS234Z027 & $2 \cdot 3$ & $14 \cdot 5$ & $0 \cdot 0235$ & $9 \cdot 5$ & $1 \cdot 079$ & $\mathrm{SF}$ \\
\hline $235 \mathrm{~s}$ & TGS235Z125 & $2 \cdot 6$ & $14 \cdot 8$ & $0 \cdot 0243$ & $9 \cdot 6$ & $0 \cdot 932$ & $\mathrm{SF}$ \\
\hline $235 \mathrm{~s}$ & XGS160Z339 & $1 \cdot 3$ & $18 \cdot 6$ & $0 \cdot 1186$ & $7 \cdot 1$ & & $\mathrm{AGN}(\mathrm{abs})$ \\
\hline $236 \mathrm{~s}$ & TGS236Z095 & $1 \cdot 8$ & $15 \cdot 4$ & $0 \cdot 0237$ & $4 \cdot 6$ & 0.558 & $\mathrm{SF}$ \\
\hline $236 \mathrm{~s}$ & TGS236Z091 & $4 \cdot 7$ & $17 \cdot 0$ & $0 \cdot 0552$ & $3 \cdot 3$ & $0 \cdot 251$ & $\mathrm{SF}$ \\
\hline $236 \mathrm{~s}$ & TGS236Z065 & $2 \cdot 3$ & $17 \cdot 6$ & $0 \cdot 1420$ & $9 \cdot 8$ & & AGN(abs) \\
\hline $236 \mathrm{~s}$ & TGS236Z194 & $4 \cdot 7$ & $15 \cdot 5$ & $0 \cdot 0237$ & $4 \cdot 0$ & $0 \cdot 490$ & $\mathrm{SF}$ \\
\hline $236 \mathrm{~s}$ & TGS236Z171 & $3 \cdot 7$ & $18 \cdot 5$ & $0 \cdot 1648$ & $7 \cdot 0$ & & AGN(abs) \\
\hline $237 \mathrm{~s}$ & TGS237Z119 & $3 \cdot 0$ & $14 \cdot 6$ & $0 \cdot 0419$ & $5 \cdot 7$ & $0 \cdot 452$ & $\mathrm{SF}$ \\
\hline $238 \mathrm{~s}$ & TGS238Z241 & $1 \cdot 6$ & $18 \cdot 2$ & $0 \cdot 2214$ & $10 \cdot 7$ & $0 \cdot 918$ & SF?(orAGN?) \\
\hline $238 \mathrm{~s}$ & TGS238Z206 & $9 \cdot 7$ & $18 \cdot 1$ & $0 \cdot 0008$ & $15 \cdot 6$ & & Galacticstar \\
\hline $238 s$ & TGS238Z047 & $3 \cdot 3$ & $16 \cdot 5$ & $0 \cdot 0215$ & $5 \cdot 3$ & $0 \cdot 741$ & $\mathrm{SF}$ \\
\hline $238 \mathrm{~s}$ & TMS238Z180 & $6 \cdot 9$ & $17 \cdot 2$ & $0 \cdot 1255$ & $3 \cdot 1$ & $0 \cdot 187$ & $\mathrm{SF}$ \\
\hline $238 \mathrm{~s}$ & TGS238Z036 & $9 \cdot 6$ & $15 \cdot 0$ & $0 \cdot 0203$ & $2 \cdot 8$ & $0 \cdot 251$ & $\mathrm{SF}$ \\
\hline $239 \mathrm{~s}$ & XGS238Z030 & $3 \cdot 1$ & $17 \cdot 2$ & $0 \cdot 0653$ & $5 \cdot 5$ & $0 \cdot 482$ & $\mathrm{SF}$ \\
\hline $239 \mathrm{~s}$ & TGS239Z196 & $8 \cdot 6$ & $14 \cdot 8$ & $0 \cdot 0219$ & $3 \cdot 5$ & $0 \cdot 301$ & SF \\
\hline $239 \mathrm{~s}$ & TGS239Z089 & $5 \cdot 4$ & $18 \cdot 8$ & $0 \cdot 2216$ & $4 \cdot 3$ & & $\mathrm{SF} ?$ \\
\hline $239 \mathrm{~s}$ & TGS239Z061 & $1 \cdot 6$ & $18 \cdot 9$ & $0 \cdot 1580$ & $3 \cdot 3$ & & AGN? \\
\hline $240 \mathrm{~s}$ & TGS240Z082 & $0 \cdot 5$ & $17 \cdot 7$ & $0 \cdot 1269$ & $11 \cdot 0$ & & $\mathrm{AGN}(\mathrm{abs}+[\mathrm{NII}] \mathrm{em})$ \\
\hline $240 \mathrm{~s}$ & TGS240Z211 & $0 \cdot 6$ & $18 \cdot 2$ & $0 \cdot 1395$ & $42 \cdot 1$ & & $\mathrm{AGN}(\mathrm{abs}+[\mathrm{NII}] \mathrm{em})$ \\
\hline $240 \mathrm{~s}$ & TGS240Z013 & $0 \cdot 4$ & $18 \cdot 9$ & $0 \cdot 2238$ & $3 \cdot 3$ & & $\mathrm{AGN}(\mathrm{abs}+[\mathrm{OIII}] \mathrm{em})$ \\
\hline $241 \mathrm{~s}$ & TGS241Z101 & $1 \cdot 8$ & $17 \cdot 8$ & $0 \cdot 1276$ & $12 \cdot 7$ & & $\operatorname{AGN}(\mathrm{abs})$ \\
\hline $313 \mathrm{~s}$ & TGS313Z081 & $4 \cdot 2$ & $17 \cdot 7$ & $0 \cdot 1220$ & $105 \cdot 8$ & & $\mathrm{AGN}(\mathrm{abs}+\mathrm{H} \alpha \mathrm{em})$ \\
\hline $313 \mathrm{~s}$ & TGS313Z100 & $7 \cdot 9$ & $17 \cdot 1$ & $0 \cdot 1474$ & $17 \cdot 0$ & & $\mathrm{AGN}(\mathrm{abs}+\mathrm{H} \alpha \mathrm{em})$ \\
\hline $314 \mathrm{~s}$ & TGS314Z122 & $0 \cdot 9$ & $17 \cdot 7$ & $0 \cdot 1440$ & $98 \cdot 1$ & & AGN(abs) \\
\hline $314 \mathrm{~s}$ & TGS314Z110 & $3 \cdot 0$ & $17 \cdot 4$ & $0 \cdot 0820$ & $5 \cdot 1$ & & AGN(abs) \\
\hline $314 \mathrm{~s}$ & TGS314Z116 & $5 \cdot 3$ & $19 \cdot 3$ & $0 \cdot 2355$ & $8 \cdot 2$ & & AGN(abs) \\
\hline $314 \mathrm{~s}$ & TMS314Z092 & $3 \cdot 1$ & $19 \cdot 2$ & $0 \cdot 2134$ & $13 \cdot 2$ & & AGN(abs) \\
\hline $318 \mathrm{~s}$ & TGS318Z046 & $5 \cdot 8$ & $18 \cdot 5$ & $0 \cdot 1544$ & $4 \cdot 9$ & & AGN(abs) \\
\hline $318 \mathrm{~s}$ & TGS318Z156 & $6 \cdot 9$ & $18 \cdot 3$ & $0 \cdot 0687$ & $2 \cdot 8$ & $0 \cdot 496$ & $\mathrm{SF}$ \\
\hline $319 \mathrm{~s}$ & TGS319Z013 & $2 \cdot 1$ & $18 \cdot 7$ & $0 \cdot 1224$ & $4 \cdot 2$ & & AGN(abs) \\
\hline $216 n$ & TGN216Z011 & $2 \cdot 2$ & $18 \cdot 8$ & $0 \cdot 1395$ & $9 \cdot 8$ & & AGN(abs) \\
\hline $216 n$ & TGN216Z148 & $6 \cdot 1$ & $17 \cdot 9$ & $0 \cdot 0832$ & $4 \cdot 1$ & & AGN(abs) \\
\hline $218 n$ & TGN218Z083 & $5 \cdot 4$ & $18 \cdot 1$ & $0 \cdot 1934$ & $2 \cdot 8$ & & $\mathrm{SF}$ \\
\hline $218 n$ & TGN218Z230 & $3 \cdot 1$ & $15 \cdot 9$ & $0 \cdot 0201$ & $3 \cdot 3$ & $0 \cdot 379$ & $\mathrm{SF}$ \\
\hline
\end{tabular}


Table 2. (Continued)

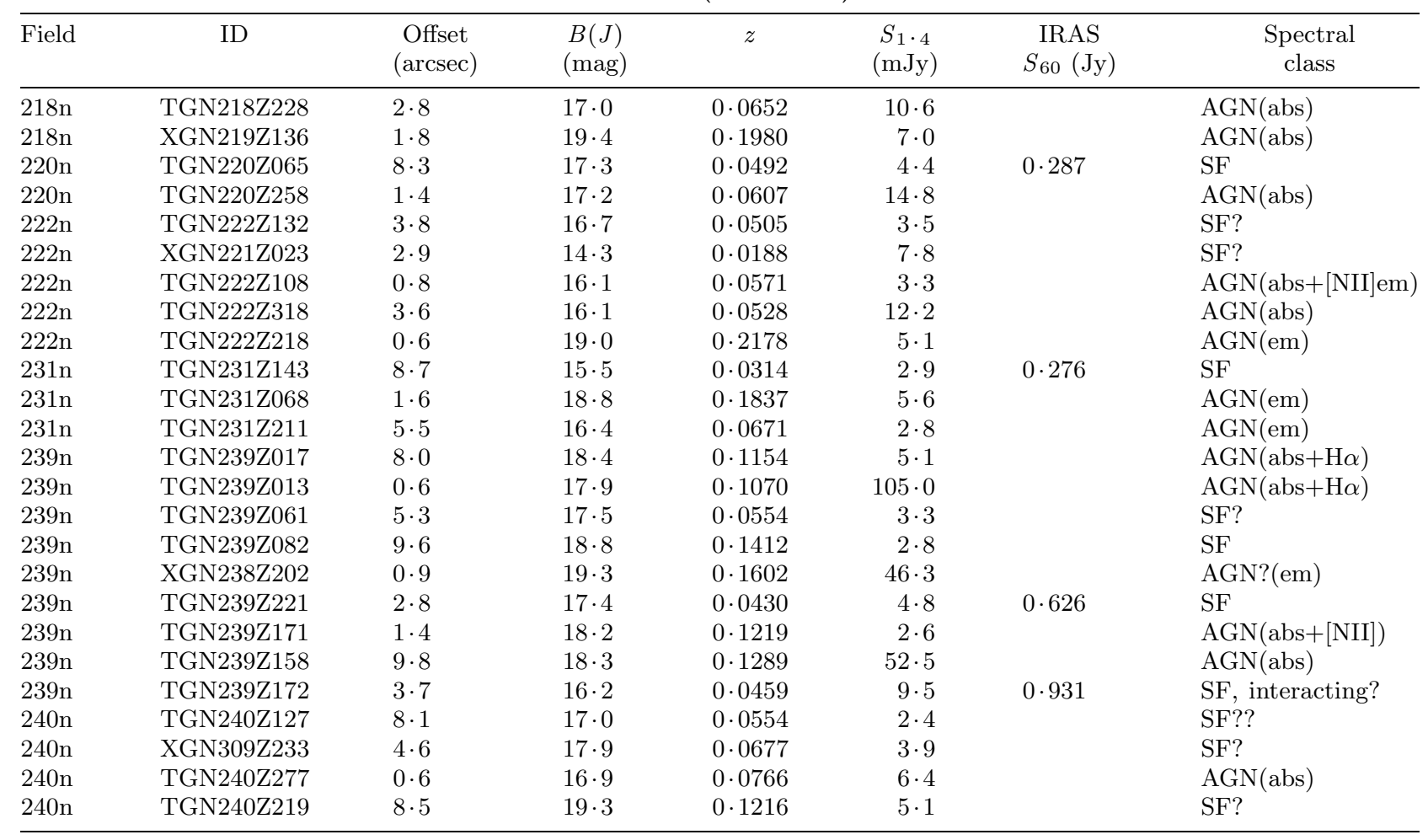

source can be identified with each optical galaxy). Thus about 27 chance coincidences are expected in a total of 8362 galaxies (see column 5 of Table 1). This calculation ignores the known clustering of galaxies on the sky, although this will only invalidate the result if there is significant clustering on scales comparable to the identification range of 15 arcsec, or if there are substantial differences between the spatial distribution of radio-loud and quiet galaxies. We believe these effects are unlikely to give an error of more than $10 \%$.

The results suggest that candidate matches with an offset of up to 10 arcsec are highly likely to be real associations. We therefore use a simple 10 arcsec cutoff in the radio-optical position difference for the analysis which follows. This gives us 99 radio-detected galaxy matches in the thirty $2 \mathrm{dF}$ fields. It also means that we have probably omitted about a dozen real identifications with larger offsets, but this is not a problem here since our aim is to make a first qualitative exploration of the faint radio galaxy population.

\section{Two Kinds of Radio Source: AGN/SF Classification}

We classified each matched galaxy as either 'AGN' or 'star-forming' (SF) based on its $2 \mathrm{dF}$ spectrum. The 'AGN' galaxies have either a pure absorption-line spectrum like that of a giant elliptical galaxy, or a stellar continuum plus nebular emission lines such as $[\mathrm{OII}]$ and $[\mathrm{OIII}]$ which are strong compared with any Balmer-line emission. Some of the emission-line AGN have spectra which resemble Seyfert galaxies.
The 'SF' galaxies are those where strong, narrow emission lines of $\mathrm{H} \alpha$ and (usually) $\mathrm{H} \beta$ dominate the spectrum. They include both nearby spirals and more distant IRAS galaxies. Figure 1 shows examples of spectra we classified as AGN, Seyfert and SF. Note that in this classification scheme, 'AGN' may simply denote the presence of radioillustrator emission, with no obvious optical signature. The origin of radio emission in the AGN and SF galaxies is believed to be quite different (e.g. Condon 1989), arising from non-thermal processes related to a central massive object in the AGN galaxies and from processes related to star formation (including supernova remnants, HII regions, etc.) in the SF galaxies.

We are confident that this simple 'eyeball' classification of the $2 \mathrm{dF}$ spectra allows us to separate the AGN and SF classes accurately. Jackson \& Londish (2000) measured several emission-line ratios (including [OIII, $\lambda 5007] / \mathrm{H} \beta,[\mathrm{NII}, \lambda 6584] / \mathrm{H} \alpha,[\mathrm{OI}$, $\lambda 6300] / \mathrm{H} \alpha$ and [SII, $\lambda \lambda 6716,6731] / \mathrm{H} \alpha$ ) for most of the galaxies studied here and plotted them on the diagnostic diagrams of Veilleux \& Osterbrock (1987). They found that the 'eyeball' classifications and line-ratio based classifications agreed more than $95 \%$ of the time, and hence that 'eyeball' classifications can be used with confidence to analyse large samples of $2 \mathrm{dF}$ spectra.

Most of the $2 \mathrm{dF}$ spectra are of impressively good quality. However, of the 98 spectra we examined (one galaxy was not actually observed by the 2dFGRS), nine had such a low signal-to-noise ratio that we were 


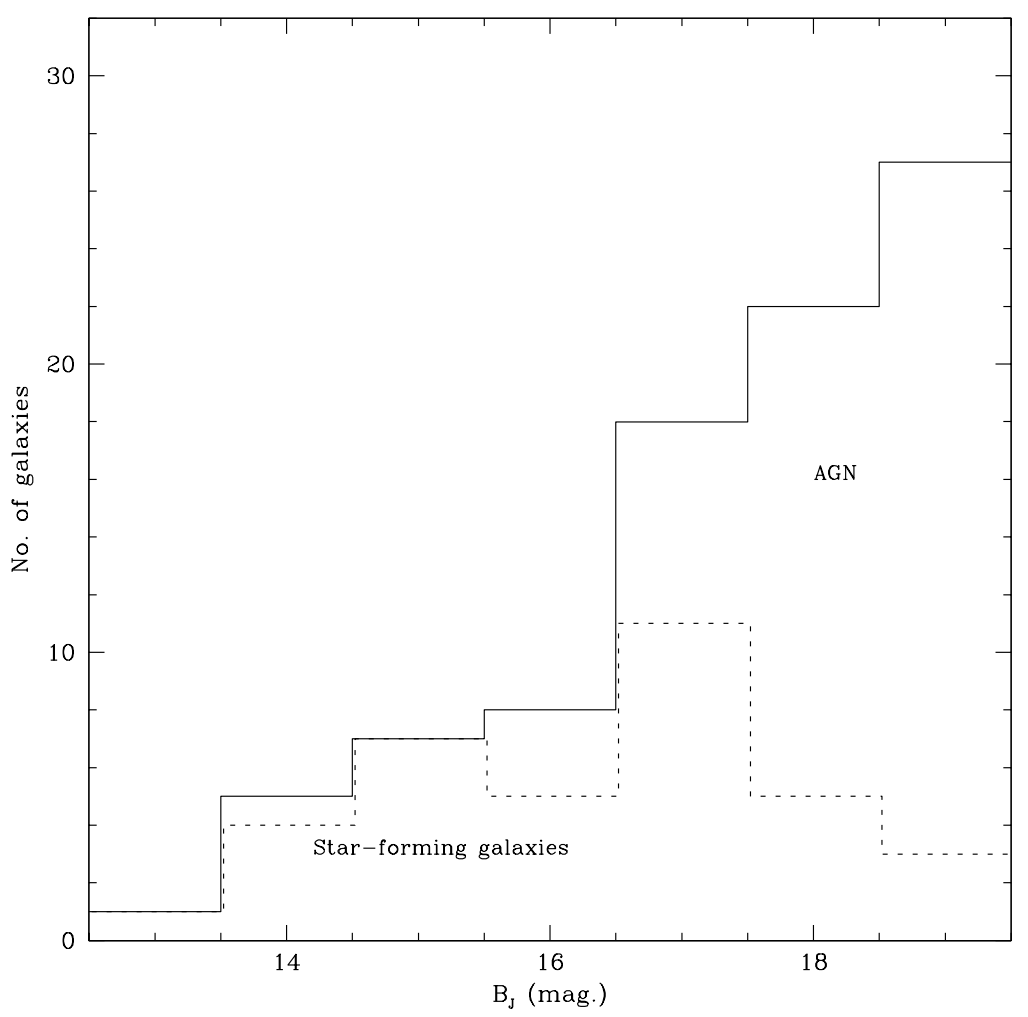

Figure 2-Distribution of the star-forming and AGN galaxies in apparent magnitude. Note that star-forming galaxies dominate at the bright end $\left(b_{\mathrm{J}}<17\right.$ mag.), while most of the fainter galaxies have AGN spectra.

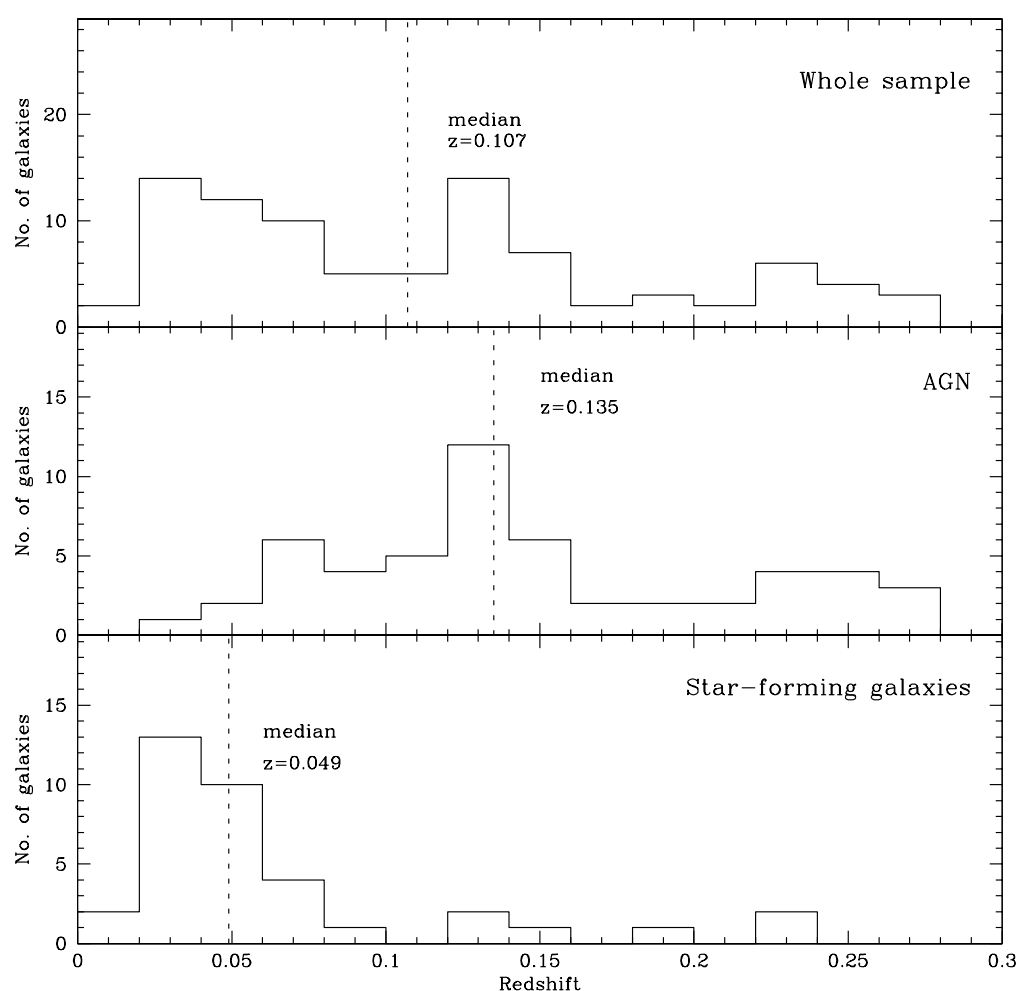

Figure 3-Redshift distribution of the AGN and star-forming galaxies, and of the whole sample. Most of the star-forming galaxies are relatively nearby, though some extend out to redshifts of $0 \cdot 25$. The AGN galaxies are a more distant population, with a median redshift almost three times that of the star-forming galaxies. 


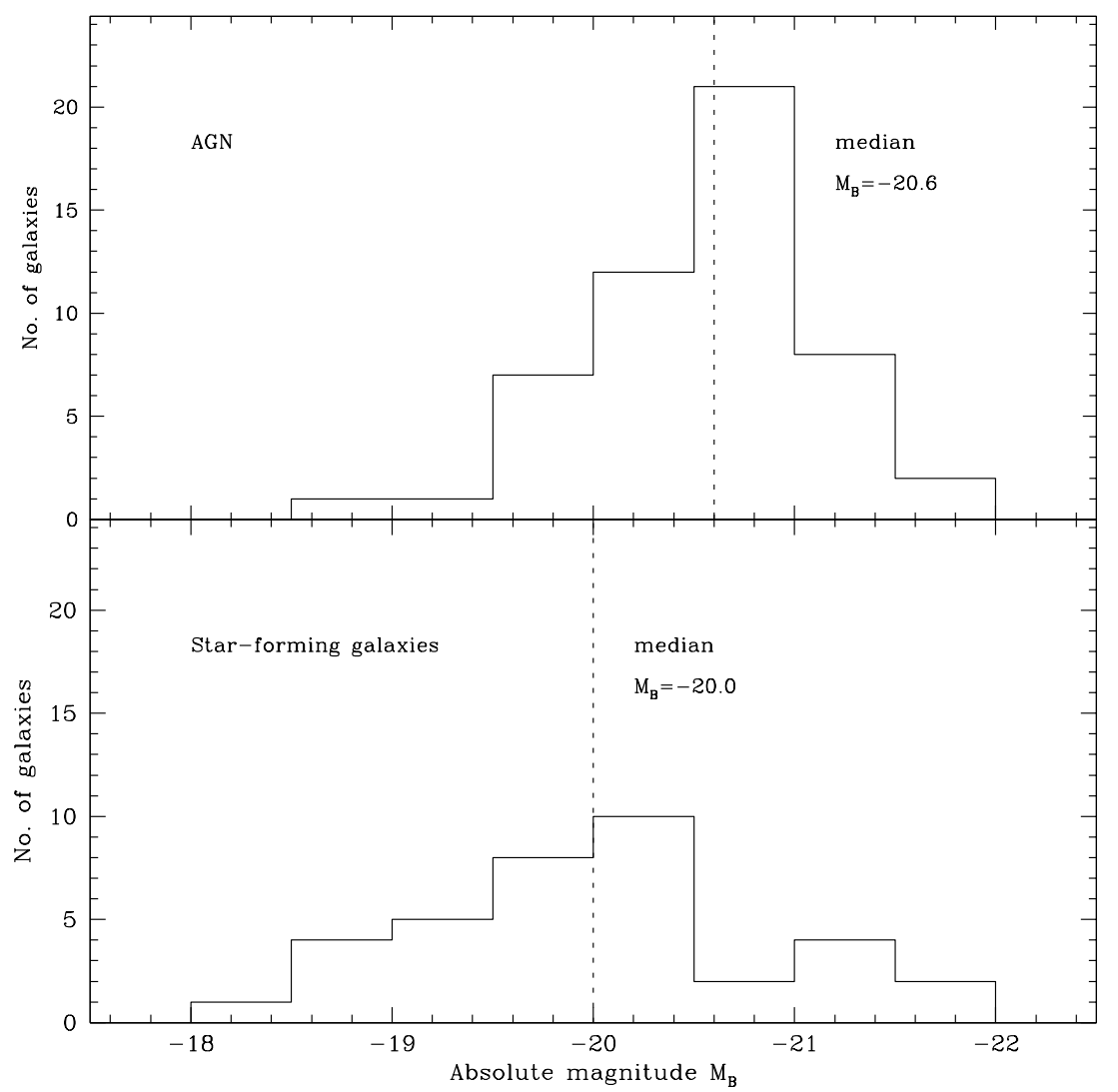

Figure 4-Absolute magnitude histograms for the AGN and star-forming galaxies. The AGN spectra are typically found in luminous optical galaxies, while the star-forming galaxies span a much wider range in optical luminosity.

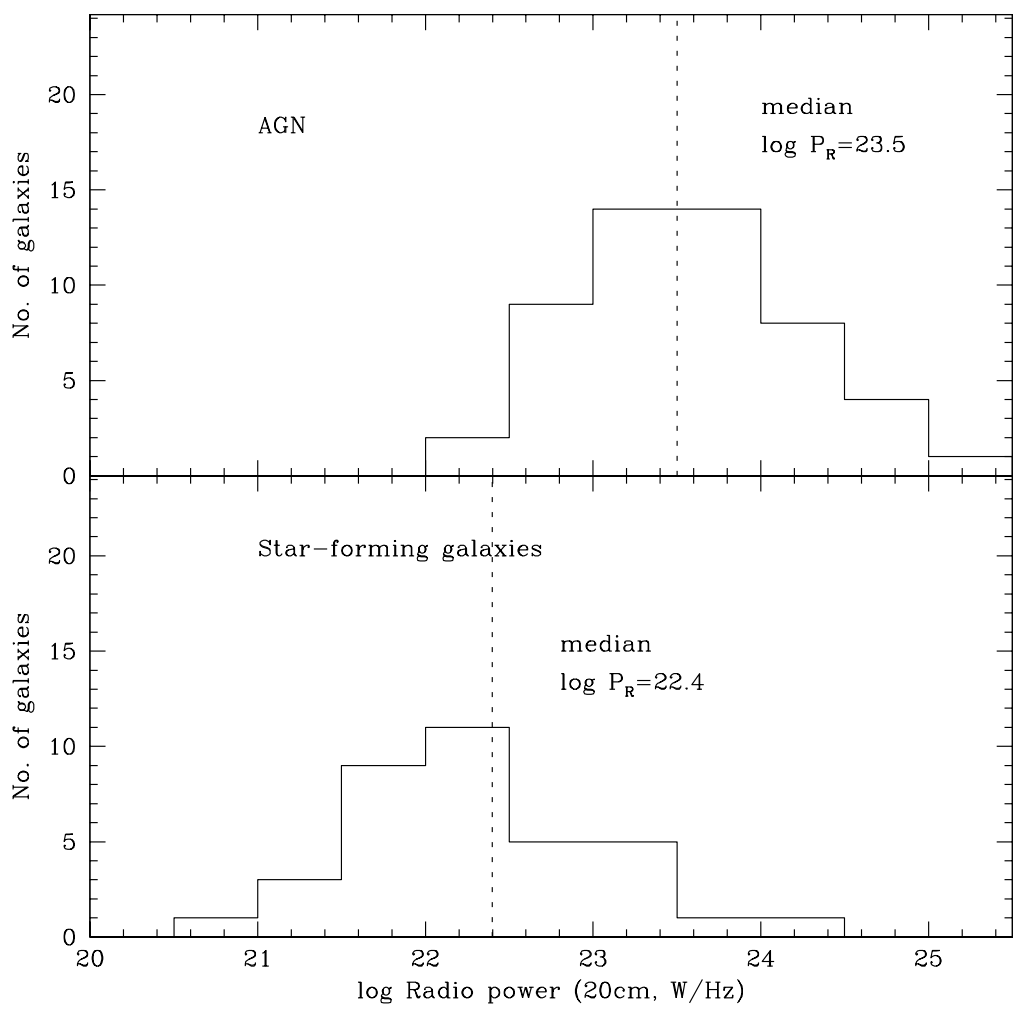

Figure 5-Histograms of radio power for the AGN and star-forming galaxies. 


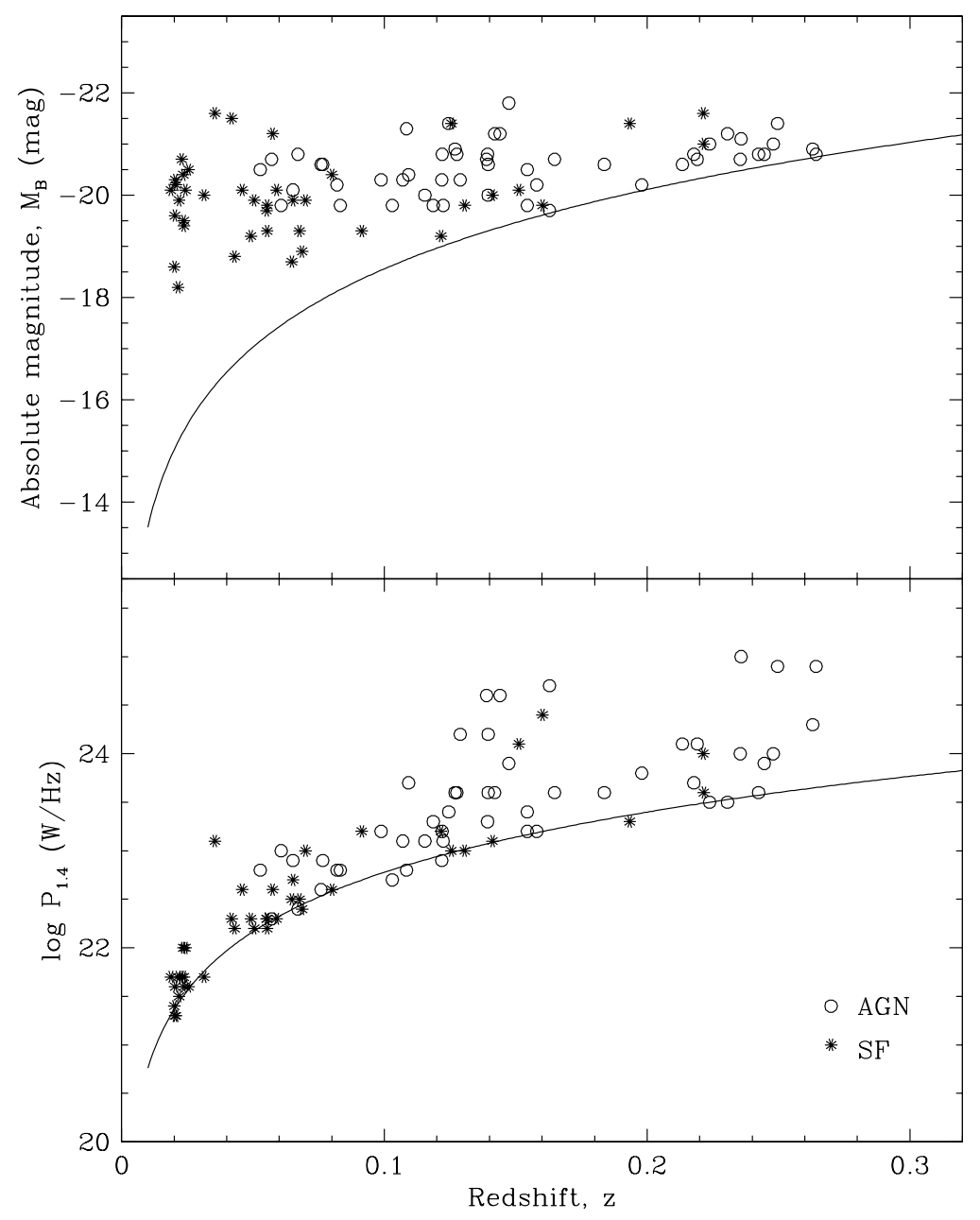

Figure 6-Plots of radio power (bottom) and optical luminosity (top) versus redshift for AGN (shown by open circles) and SF (shown by stars) galaxies. The curve in each plot shows the radio and optical limits of the NVSS and 2dFGRS respectively-galaxies below these curves will drop out of our sample.

unable to classify the spectrum. One other object appeared to be a Galactic star. We were therefore left with 88 good-quality $2 \mathrm{dF}$ spectra of candidate radio matches with an offset $D<10$ arcsec. Of these 88 galaxies, $36(41 \%)$ were classified as $\mathrm{SF}$ and 52 $(59 \%)$ as AGN. One galaxy classified as SF had an emission-line spectrum which resembled an AGN, but was also detected as an IRAS source at $60 \mu \mathrm{m}$. This may be a genuinely composite object. Table 2 lists the matched galaxies, their spectral classification, 1.4 GHz radio continuum flux density, apparent magnitude and redshift. A more quantitative spectral classification using diagnostic emission-line ratios will be presented in the forthcoming paper by Jackson \& Londish (2000).

Figures 2 and 3 show the distribution of AGN and SF classes in apparent magnitude and redshift respectively. There is a clear segregation in apparent magnitude: most galaxies brighter than about $b_{\mathrm{J}}$ $=16 \cdot 5-17$ mag. fall into the star-forming $(\mathrm{SF})$ class, while the AGN class dominates the population fainter than $b_{\mathrm{J}} \sim 17$. This reflects strong differences in the global properties of the two classes as well as the radio and optical flux limits of the NVSS and 2dFGRS. The AGN galaxies are typically more distant than the SF galaxies (by about a factor of 3 - see Figure 3), and more luminous both optically and in radio power (see Figures 4 and 5). We know that the SF galaxies continue to large redshifts and to very faint optical magnitudes (e.g. Benn et al. 1993), but these galaxies quickly drop out of our sample because of the 2-3 mJy limit of the NVSS in radio flux density. Similarly, we know that the AGN galaxies extend to much higher redshifts than probed by the 2dFGRS, but these distant AGN galaxies will be fainter than the $b_{\mathrm{J}}=19.4 \mathrm{mag}$. optical limit of the 2dFGRS. Figure 6 shows plots of radio power and optical luminosity versus redshift for the AGN and SF classes - the curves correspond to the survey completeness limits of $3.5 \mathrm{mJy}$ and 19.4 mag. for the NVSS and 2dFGRS respectively. Galaxies below these lines will be excluded from our sample. Note that most of the SF galaxies are weak radio sources, lying close to the NVSS cutoff 


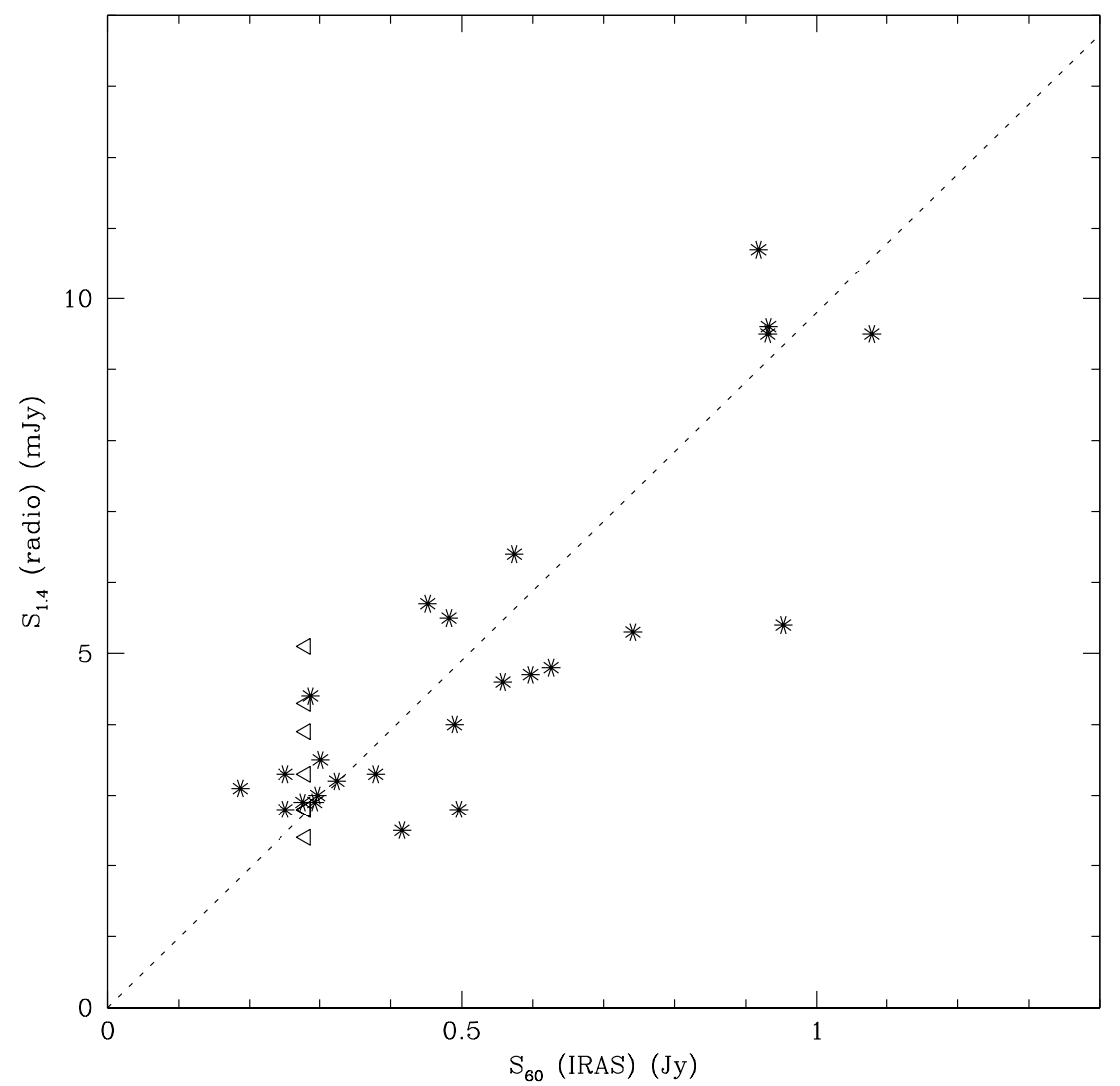

Figure 7-Comparison of radio $(1.4 \mathrm{GHz})$ and IRAS far-infrared $(60 \mu \mathrm{m})$ flux density for the radio-detected star-forming galaxies in the 2dFGRS. The dotted line is for $S_{60 \mu \mathrm{m}}=100 S_{1} \cdot 4 \mathrm{GHz}$. Of the nine SF galaxies in Table 2 which lack IRAS data, two lie in regions not observed by IRAS. For the remaining seven we show (open triangles) upper limits of $0 \cdot 28 \mathrm{Jy}$ at $60 \mu \mathrm{m}$, corresponding to the limits of the IRAS Faint Source Catalogue.

at all redshifts, while most of the AGN galaxies lie well above the radio limit but start to drop below the optical cutoff at redshifts above $0 \cdot 15$.

\section{Matches with IRAS Sources}

We expect many of the SF radio sources to be IRAS detections, based on the well-known correlation between radio continuum and far-infrared (FIR) luminosities (e.g. Wunderlich, Klein \& Wielebinski 1987; Condon, Anderson \& Helou 1991). For spiral galaxies, $S_{60 \mu \mathrm{m}} \sim 100 S_{1} \cdot 4 \mathrm{GHz}$ (e.g. Condon \& Broderick 1988; Rowan-Robinson et al. 1993), so NVSS should detect most galaxies in the IRAS Faint Source Catalog (which has a flux density limit of $0 \cdot 28 \mathrm{Jy}$ at $60 \mu \mathrm{m})$.

Of the 36 galaxies classified as SF in Table 2, two (TGN222Z132 and XGN221Z023) lie in the 3\% of the sky which has no IRAS coverage (Beichman et al. 1985). Of the remaining 34 galaxies, 27 (i.e. 79\%) are detected at $60 \mu \mathrm{m}$ in the IRAS Point Source Catalog or Faint Source Catalog (FSC). Figure 7 compares the radio continuum $(1 \cdot 4 \mathrm{GHz})$ and IRAS $(60 \mu \mathrm{m})$ flux densities for these galaxies (for galaxies undetected by IRAS we show an upper limit of $0 \cdot 28$ Jy, corresponding to the completeness level of the FSC). If we exclude one galaxy with anomalously strong $60 \mu \mathrm{m}$ emission as discussed below, the mean FIR-radio ratio $Q_{60}=S_{60 \mu m} / S_{1} \cdot 4 \mathrm{GHz}$ for the IRAS-detected galaxies is $112 \pm 8$, i.e. close to that derived from other studies.

One galaxy (TMS206Z015) has an unusually high value of $Q_{60}=380$, with much stronger FIR emission than would be expected from the radio continuum flux density. The most likely explanation is confusion in the IRAS beam, since this galaxy lies in a group and appears to be interacting with a companion.

\section{Conclusions}

Based on a study of thirty fields from the $2 \mathrm{dF}$ Galaxy Redshift Survey, we find that about $1.5 \%$ of 2 dFGRS galaxies brighter than $b_{\mathrm{J}}<19 \cdot 4$ magnitude are candidate identifications for $1 \cdot 4 \mathrm{GHz}$ NVSS radio sources. Of these about $80-85 \%$ will turn out, after closer examination, to be 'real' associations. Thus if the whole 2dFGRS contains 250,000 galaxies, we expect to identify about 4000 candidate matches with NVSS by the time the 2dFGRS is complete. About $60 \%$ of these galaxies will be AGN (radio galaxies and some Seyferts) and $40 \%$ star-forming galaxies. For galaxies south of declination $-30^{\circ}$, we will also have $843 \mathrm{MHz}$ radio flux density measurements from the Sydney University Molonglo Sky Survey 
(SUMSS; Bock, Large \& Sadler 1999), and will be able to measure radio spectral indices.

The final sample will be by far the largest (and most homogeneous) sample of radio-galaxy spectra ever obtained, and will allow us to study both the AGN and starburst radio populations out to a redshift $z \sim 0 \cdot 3$, and to look for evidence of evolution over this redshift range. As the present paper goes to press, the $2 \mathrm{dF}$ Galaxy Redshift Survey is $20 \%$ complete and our sample has already grown to more than 700 galaxies. This larger sample will be analysed in more detail in a forthcoming paper.

\section{Acknowledgments}

We thank the 2dF Galaxy Redshift Survey team for allowing us early access to their data. We also acknowledge the essential contribution of the many people, at the AAO and elsewhere, who have contributed to the building and operation of the $2 \mathrm{dF}$ facility. Finally, we thank the two anonymous referees of this paper for their careful reading and helpful suggestions.

The IRAS flux densities quoted in Table 2 were obtained from the NASA Extragalactic Database (NED).

\section{References}

Becker, R. H., White, R. L., \& Helfand, D. J. 1995, ApJ, 450, 599

Beichman, C. A., Neugebauer, G., Habing, H. J., Clegg, P. E., \& Chester, T. J. 1985, IRAS Explanatory Supplement, JPL
Benn, C. R., Rowan-Robinson, M., McMahon, R. G., Broadhurst, T. J., \& Lawrence, A. 1993, MNRAS, 263, 98

Bock, D. C.-J., Large, M. I., \& Sadler, E. M. 1999, AJ, 117, 1578

Colless, M. 1999, Phil. Trans. R. Soc. London A, 357, 105 (astro-ph/9804078)

Condon, J. J. 1984, ApJ, 287, 461

Condon, J. J. 1989, ApJ, 338, 13

Condon, J. J. 1992, ARA \& A, 30, 575

Condon, J. J., \& Broderick, J. J. 1988, AJ, 96, 30

Condon, J. J., Anderson, M. L., \& Helou, G. 1991, ApJ, 376,95

Condon, J. J., Cotton, W. D., Greisen, E. W., Yin, Q. F., Perley, R. A., Taylor, G. B., \& Broderick, J. J. 1998, AJ, 115, 1693

Cress, C. M., Helfand, D. J., Becker, R. H., Gregg, M. D., \& White, R. L. 1996, ApJ, 473, 7

Folkes, S., et al. 1999, MNRAS, 308, 459

Jackson, C. A., \& Londish, D. M. 2000, PASA, submitted

Lewis, I. J., Glazebrook, K., \& Taylor, K. 1998, SPIE Proc., 3355,828

Maddox, S. J. 1998, in Large Scale Structure: Tracks and Traces, Proc. 12th Potsdam Cosmology Workshop, ed. V. Müller et al. (Singapore: World Scientific), p. 91 (astro-ph/9711015)

Magliocchetti, M., Maddox, S., Lahav, O., \& Wall, J. V. 1998, MNRAS, 300, 257

Rengelink, R. B., Tang, Y., de Bruyn, A. G., Miley, G. K., Bremer, M. N., Röttgering, H., \& Bremer, M. A. R. 1997, A\&AS, 124, 259

Rowan-Robinson, M., Benn, C. R., Lawrence, A., McMahon, R. G., \& Broadhurst, T. J. 1993, MNRAS, 263, 123

Smith, G., \& Lankshear, A. 1998, SPIE Proc., 3355, 905

Veilleux, S., \& Osterbrock, D. A., 1987, ApJS, 63, 295

Windhorst, R. A., Miley, G. K., Owen, F. N., Kron, R. G., \& Koo, D. C. 1985, ApJ, 289, 494

Wunderlich, E., Klein, U., \& Wielebinski, R. 1987, A\&AS, 69,487 